\title{
Diabetes mellitus tipo 2 y cardiopatía isquémica: fisiopatología, regulación génica y futuras opciones terapéuticas
}

\author{
Larissa Aleman ${ }^{1^{\star}}$, Andrea Ramírez-Sagredo ${ }^{1^{\star}}$, Jafet Ortiz-Quintero ${ }^{1}$, Sergio Lavandero ${ }^{1,2}$ \\ 1 Advanced Center for Chronic Diseases (ACCDiS) \& Centro Estudios Moleculares de la Célula (CEMC). Facultad de Ciencias \\ Químicas y Farmacéuticas y Facultad de Medicina, Universidad de Chile, Santiago, Chile. \\ 2 Cardiology Division, Department of Internal Medicine, University of Texas Southwestern Medical Center, Dallas, Texas, USA.
}

${ }^{*}$ Ambas son primeras autoras y contribuyen de igual manera a este manuscrito.

En los últimos años, la diabetes mellitus tipo 2 (DM2) ha evolucionado en forma epidémica, experimentando un rápido crecimiento y afectando a millones de individuos a nivel mundial. La cardiopatía isquémica es la principal causa de mortalidad en los pacientes diabéticos, quienes poseen un mayor riesgo cardiovascular respecto a los no diabéticos. La DM2 y la cardiopatía isquémica se caracterizan por ser prevenibles, sin embargo, existen diversos factores de riesgo comunes que contribuyen a su desarrollo.

Los mecanismos que explican la ateroesclerosis acelerada y el incremento de riesgo de enfermedades cardiovasculares en los pacientes diabéticos tipo 2 incluyen a la hiperglicemia, dislipidemia y la inflamación del endotelio vascular.

La diabetes es resultado de una interacción compleja entre la genética y el medio ambiente. Recientemente se han descrito varios genes implicados en el desarrollo de la diabetes y cardiopatía isquémica y que podrían significar nuevas opciones terapéuticas.

En este artículo se revisa la relación entre ambas patologías, los mecanismos moleculares y el descubrimiento de factores de riesgo genéticos comunes y su implicancia en el desarrollo de nuevos blancos terapéuticos. Palabras Clave: Diabetes mellitus, cardiopatía isquémica, genética. 


\section{Type II Diabetes and Ischemic Heart disease: pathophysiology, gen regulation and future therapeutic options}

In recent years, type 2 diabetes mellitus has evolved as a rapidly increasing epidemic and affects millions of people worldwide. Ischemic heart disease (IHD) is the main cause of death among diabetic patients, who have a higher cardiovascular risk than non-diabetics. Both, DM2 and IHD are characterized by being preventable, however there are several common risk factors that contribute to their development.

The mechanisms that explain accelerated atherosclerosis and increased risk of cardiovascular diseases in patients with type 2 diabetes mellitus include damage by hyperglycemia, dyslipidemia and inflammation on vascular endothelium.
Diabetes is the result of a complex interaction between genetics and the environment, recently, several genes have been identified that appear to be involved in diabetes and ischemic heart disease that could explain its relationship and serve as new therapeutic possibilities.

In this article, we review the relationship between diabetes and ischemic heart disease, the molecular mechanisms and the discovery of genetic risk factors common to both diseases and their implication in the development of new therapeutic targets.

Key Words: Diabetes, ischemic heart disease, genetics. 


\section{Introducción:}

La diabetes mellitus (DM) y la cardiopatía isquémica constituyen una epidemia global, representando una gran carga para la salud pública. El número de diabéticos crece de forma alarmante; estimándose que afecta a más de 422 millones de personas en todo el mundo. Para el año 2035 se proyecta 592 millones de diabéticos ${ }^{1,2}$. La DM es un grupo de enfermedades metabólicas caracterizada por hiperglicemia, producto de defectos en la producción, secreción y/o acción de la insulina. Se sub-clasifican en: DM tipo 1 (DM1), DM tipo 2 (DM2), diabetes gestacional (DMG) y otros tipos específicos ${ }^{3}$. Los pacientes con DM2 suponen la mayor proporción de personas que padecen esta patología.

La cardiopatía isquémica, la principal causa de morbimortalidad a nivel mundial, se caracteriza por la reducción del flujo sanguíneo a regiones del miocardio. Los pacientes con DM2 tienen mayor morbilidad y mortalidad cardiovascular en comparación con sujetos no diabé$\operatorname{ticos}^{4-6}$. Ambas patologías se caracterizan por ser prevenibles, sin embargo, existen diversos factores de riesgo comunes que contribuyen a su desarrollo. Se han logrado grandes avances en la descripción de los mecanismos fisiopatológicos que producen ambas enfermedades, sin embargo, aún existen aspectos por dilucidar. En el desarrollo de ambas patologías existe una compleja interacción entre la genética y el medio ambiente. La alta prevalencia de pacientes que padecen simultáneamente de diabetes y cardiopatía isquémica ha llevado a la hipótesis de un efector común. En este artículo se revisa la relación entre la DM2 y la cardiopatía isquémica, discutiremos los mecanismos moleculares implicados, los nuevos descubrimientos entorno al papel de la genética en ambas enfermedades y nuevas perspectivas hacia el futuro.

\section{Diabetes mellitus y cardiopatía isquémica}

Estudios epidemiológicos han mostrado de manera consistente que individuos con DM2 tienen el doble de riesgo de desarrollar enfermedad coronaria, en comparación con individuos sanos ${ }^{9-11}$. Aunque se observa una asociación consistente entre el control glicémico y la enfermedad cardiovascular, el efecto del control glicémico estricto parece no reducir el riesgo cardiovascular en estos pacientes. Este hallazgo se ejemplificó en el estudio ACCORD (Action to Control Cardiovascular Risk in Diabetes), en el cual hubo un control glicémico intensivo, sin embargo, debió ser suspendido debido a un aumento en el número de muertes cardiovasculares ${ }^{13}$. Por otra parte, el estudio clínico ADVANCE (Action in Diabetes and
Vascular Disease) permitió descartar la presión arterial como marcador inicial en el diagnóstico de cardiopatía isquémica en pacientes con DM2, estableciendo al riesgo vascular como parámetro valioso y determinante de los protocolos de tratamiento apropiados en estos pacientes $^{16,17 .}$

La emisión de informes detallados y explicativos sobre los resultados obtenidos de estos ensayos clínicos se dificulta, ya que los mecanismos responsables del alto riesgo de enfermedad cardiovascular que acompaña a la DM2 y posiblemente al metabolismo alterado de la glucosa siguen siendo poco conocidos ${ }^{7}$. Como se mencionó anteriormente, existe fuerte evidencia de que los factores de riesgo convencionales, como la hipertensión arterial, la obesidad y la dislipidemia, no explican completamente el alto riesgo de enfermedad cardiovascular ${ }^{16}$. Sin embargo, resultados de estudios básicos in vitro, en modelos experimentales y en pacientes diabéticos sugieren varios mecanismos que apuntan a que la hiperglicemia podría afectar la aterogénesis a nivel de la pared arterial ${ }^{7,11,20}$

En la patogenia de los eventos cardiovasculares, se ha evidenciado que los factores de riesgo poseen un efecto independiente y no sinérgico. ${ }^{7-9}$ Sin embargo, se ha postulado que las personas con DM2 son propensas a las consecuencias asociadas a la disfunción endotelial. Esto implicaría un efecto potenciador entre DM2 y disfunción endotelial en la génesis y desarrollo de la cardiopatía isquémica9 ${ }^{9-23}$ (Figura 1).

Desde un punto de vista clínico, la detección de la interacción entre los factores de riesgo es importante porque identifica los objetivos terapéuticos clave, por tanto, las intervenciones dirigidas a dichos factores de riesgo son potencialmente más eficaces que el tratamiento de los factores de riesgo que no interactúan $9,11,16$.

En consecuencia, puede existir un círculo vicioso entre la disfunción endotelial y la $\mathrm{DM}^{29,23}$. El mecanismo que podría explicar a este fenómeno es una asociación bidireccional entre la disfunción endotelial y la DM2, en la que la disfunción endotelial puede actuar como causa y también como consecuencia de $\mathrm{DM}^{27,23}$. Por un lado, la DM2 conduce a la disfunción endotelial mediante, la formación de productos finales glicosilados, la acumulación intraendotelial de glucosa y el aumento del estrés oxidativo; y por otro lado, la disfunción endotelial causa o agrava la DM2 al deteriorar el acceso oportuno de glucosa e insulina a sus tejidos blanco ${ }^{11,23,30}$ (Figura 1).

\section{Mecanismos moleculares de la cardiopatía isquémica en el paciente diabético}




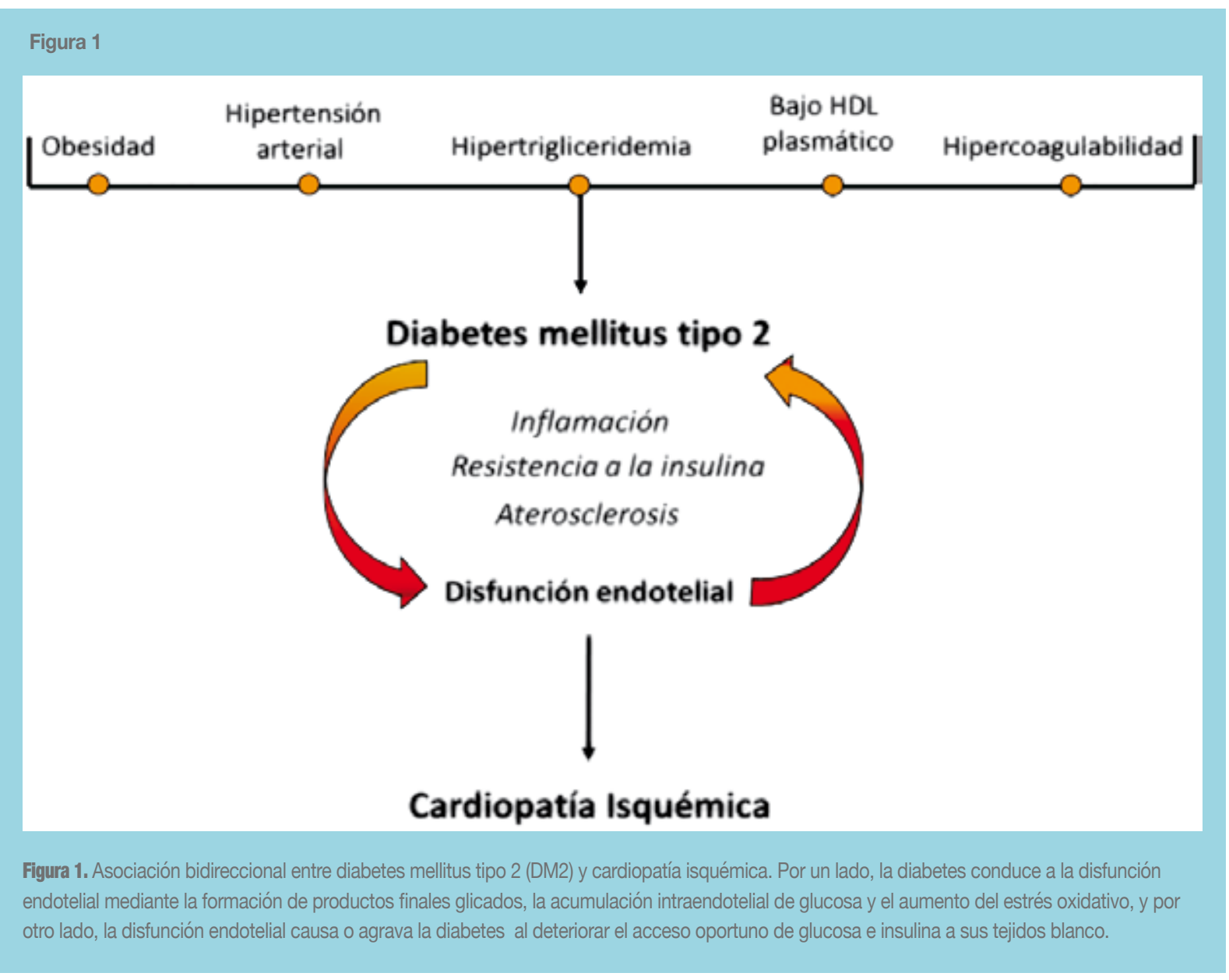

Se han descrito múltiples mecanismos que explican una ateroesclerosis acelerada y el incremento de riesgo de enfermedades cardiovasculares en pacientes con DM2. A continuación, se revisa el efecto de la hiperglicemia, dislipidemia y la inflamación en el daño vascular acelerado y el riesgo de enfermedades cardiovasculares en pacientes diabéticos.

\section{Efectos de la hiperglicemia en pacientes diabéticos}

La hiperglicemia puede provocar complicaciones vasculares por diferentes mecanismos. Las altas concentraciones de glucosa pueden activar al factor transcripcional NF- kB, el cual puede desencadenar la expresión de varios genes en células endoteliales, macrófagos derivados de monocitos y células de músculo liso vascular ${ }^{7}$. La activación del factor transcripcional NF-kB promueve vías pro-inflamatorias y puede ser activado por el receptor de reconocimiento de patrones y/o por el receptor para productos finales de glicación avanzada (RAGE).

Los productos finales de glicación avanzada (AGEs) son un grupo heterogéneo de moléculas generadas por medio de reacciones no enzimáticas de glicación de proteínas, lípidos y ácidos nucleicos con azúcares reductores (p.ej. glucosa) para formar productos de glicación temprana, también conocidos como productos de Amadori (Figura 2). Los productos de glicación temprana posteriormente se oxidan, deshidratan y entrecruzan para generar los diversos $\mathrm{AGEs}^{8}$. Una mayor formación de AGEs ocurre en condiciones, tales como la DM y el envejecimiento.

Hay evidencia que muestra que los AGEs contribuyen al desarrollo y progresión de disfunción cardiovascular mediante tres mecanismos básicos: 1) Entrecruzamiento con proteínas de la matriz extracelular, afectando las propiedades mecánicas de los tejidos, 2) Entrecruzamiento con proteínas intracelulares, alterando sus funciones fisiológicas, y 3) Unión a sus receptores de superficie RAGE para inducir múltiples cascadas de señalización ${ }^{12}$.

Por otro lado, la glucosa aumenta el estrés oxidativo, ocasionando diversos efectos lesivos en la pared arterial. La auto-oxidación de la glucosa estimula la formación 


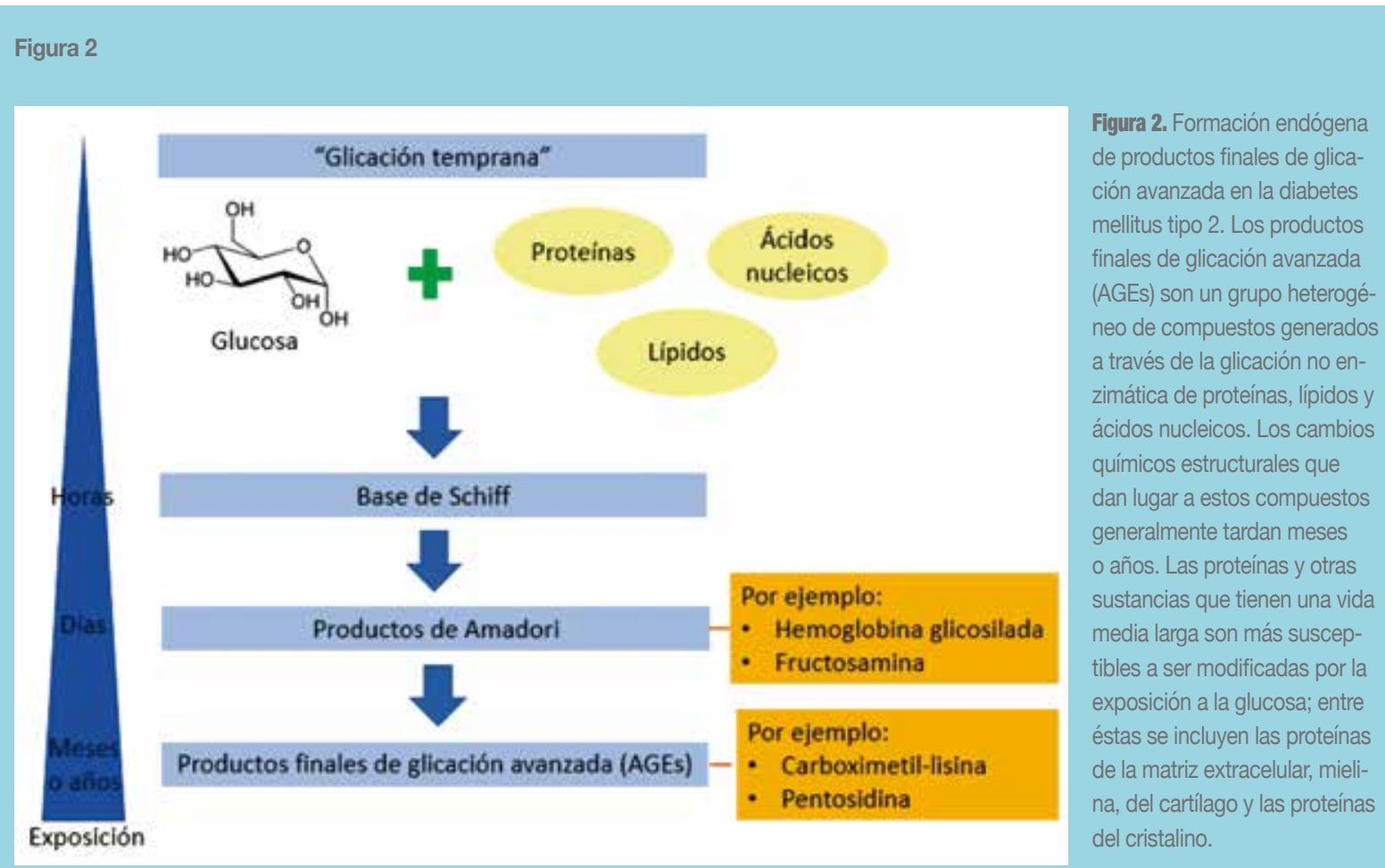

de diversas especies reactivas del oxígeno (ROS) como el anión superóxido, el cual promueve la oxidación de las lipoproteínas de baja densidad (LDL). Hay evidencias que muestran un aumento en la oxidación de las lipoproteínas en los pacientes con DM2, el cual se correlaciona con un control glicémico inadecuado. Las reacciones de glico-oxidación contribuyen a la enfermedad macrovascular en pacientes diabéticos al dañar tejidos en el microambiente local de la pared arterial. Las vías que conducen a estas reacciones incluyen la generación del anión superóxido en la mitocondria, generación de NADPH en los macrófagos, o un mecanismo sensible al estrés oxidativo que genera radicales hidroxilo ${ }^{7}$.

Estudios experimentales muestran que la hiperglicemia favorece la adhesión de monocitos a las células endoteliales por activación del factor transcripcional NF-kB, quien incrementa la expresión de diversos genes asociados a inflamación, incluyendo moléculas de adhesión que promueven la adhesión de los monocitos a las células endoteliales.

La expresión de estas moléculas de adhesión pueden ser el resultado de un desbalance en la producción de óxido nítrico (NO), debido a que los factores que incrementan la producción de NO inhiben la síntesis de estas moléculas de adhesión ${ }^{14,15}$. La inhibición de la producción de
NO por las células endoteliales mediada por la hiperglicemia y los AGEs se asocia a un desbalance de la vasodilatación dependiente del endotelio, un marcador temprano de daño vascular ${ }^{7,18}$. La hiperglicemia y los AGEs estimulan la producción de superóxido en las células endoteliales, en parte por la activación de la NADPH oxidasa ${ }^{19}$, lo cual provee una conexión entre hiperglicemia, AGEs y estrés oxidativo ${ }^{14}$.

Tanto la hiperglicemia como los AGEs se asocian con el incremento en la activación de los monocitos circulantes. Los monocitos cultivados en altas concentraciones de glucosa o aquellos aislados de pacientes con pobre control glicémico se encuentran en un estado activado e inflamatorio, lo que se evidencia con la sobreexpresión de citoquinas como interleukinas (IL) $1 \beta$ e IL $^{6}$ junto a la expresión de moléculas quimoatractantes de monocitos $^{21,22}$. Estos cambios inflamatorios se asocian con la activación de la proteína kinasa $\mathrm{C}$ (PKC), activación del NF-kB y el aumento de la generación de superóxido, los tres son responsables del estrés oxidativo que ocurre en presencia de la hiperglicemia. Los monocitos que ingresan al espacio endotelial en respuesta a factores quimotácticos, proliferan y se diferencian a macrófagos, los cuales se acumulan en la pared arterial. No obstante, la hiperglicemia no es suficiente para estimular la prolife- 


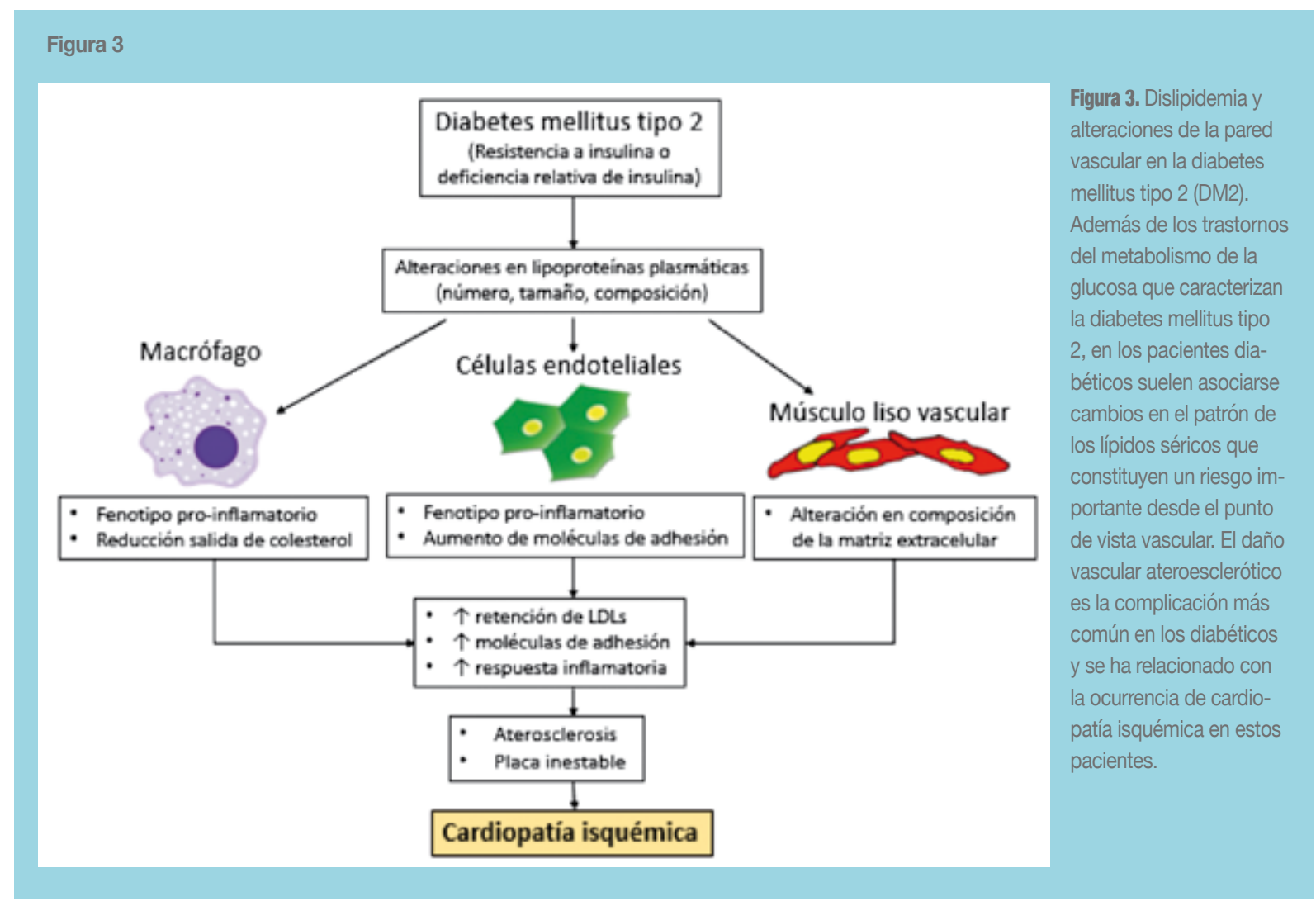

ración de los macrófagos, en combinación con hiperlipidemia se estimula la proliferación de los macrófagos por una vía que involucra la oxidación de las LDL dependiente de glucosa.

Estudios in vitro muestran que las altas concentraciones de glucosa estimulan la proliferación de células del músculo liso vascular. A medida que las lesiones ateroescleróticas progresan, las células del músculo liso vascular migran de la media a la íntima, donde proliferan, generan factores de crecimiento y participan en la formación de la capa fibrosa. Se observan resultados similares con la exposición a AGEs y altas concentraciones de insulina, que frecuentemente acompañan a la hiperglicemia en pacientes con DM2. Así mismo, la hiperglicemia se asocia con la generación de proteoglicanos, pérdida del contenido de elastina en la íntima e incremento en la fragmentación de la elastina, lo que conduce a un incremento en la retención de LDL en la pared arterial contribuyendo de esa forma a la ateroesclerosis. La retención de LDL también se favorece con la acumulación de colágeno sintetizado por las células del músculo liso vascular, el cual en presencia de hiperglicemia sufre reacciones no enzimáticas de glicación, lo que mejora su unión a las $\mathrm{LDL}^{7}$.

Efectos de la dislipidemia en pacientes diabéticos
La dislipidemia observada en los pacientes diabéticos está fuertemente relacionada con la ateroesclerosis. La DM2 se caracteriza por aumentos en las concentraciones del colesterol LDL y de las lipoproteínas VLDL y quilomicrones, disminución de la concentración del colesterol HDL y alteraciones en la composición de las lipoproteínas. Las VLDL y quilomicrones aumentan en estados de ayuno o postprandial en pacientes con DM2. El papel de estas lipoproteínas en el desarrollo de la ateroesclerosis en pacientes diabéticos es aún controversial ${ }^{7}$. Sin embargo, evidencia in vitro apoyan su efecto pro aterogénico en la pared vascular. Las lipoproteínas enriquecidas en triglicéridos favorecen el fenotipo pro-inflamatorio de las células endoteliales y macrófagos e inducen apoptosis en las células endoteliales y acumulación de lípidos en los macrófagos ${ }^{24}$. Además, estas lipoproteínas inducen la sobreexpresión de TNF- $\alpha$ y receptores de adhesión en macrófagos, lo que conduce a una mayor adherencia de monocitos a las células endoteliales ${ }^{25}$ (Figura 3).

Está bien establecido que el colesterol de las lipoproteínas de baja densidad (LDL-C) es un factor de riesgo de enfermedades cardiovasculares ${ }^{26}$. Los pacientes con DM2 podrían no tener concentraciones de LDL-C sustancialmente mayores que los individuos sanos, pero 


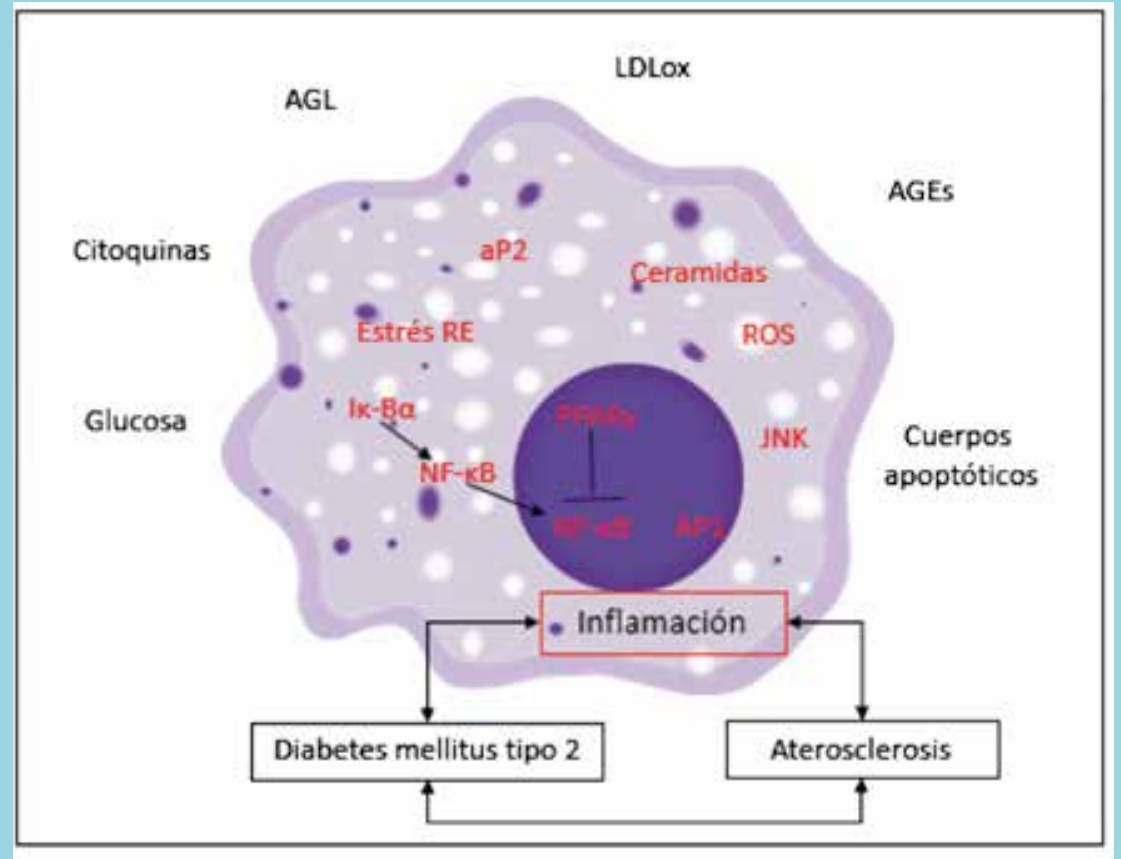

Figura 4. Efectos de los macrófagos en la diabetes mellitus tipo 2 y la aterosclerosis. Diversos estímulos y vías de señalización celular están implicados en los efectos de los macrófagos, incluyendo el aumento de la formación de células espumosas y la secreción de factores de crecimiento y citoquinas pro-inflamatorias. Estos efectos enfatizan la conexión importante entre la resistencia a la insulina, inflamación y ateroesclerosis. AGEs: productos finales de glicación avanzada, AGL: ácidos grasos libres, AP1: proteína activadora 1, aP2: proteína 2 del adipocito, Ik-Ba: inhibidor kBa, JNK: c-jun N terminal kinasa, LDLox: lipoproteínas de baja densidad oxidadas, NF-kB: factor nuclear KB, PPARy: receptor de peroxisoma-proliferador-activado gamma, RE: retículo endoplásmico, ROS: especies reactivas del oxígeno.

para cualquier concentración de LDL-C, los pacientes diabéticos, generalmente, presentan un aumento en las partículas de LDL, usualmente más pequeñas, densas, pobres en lípidos y que tienden a acumularse en la circulación. Además, presentarán altas concentraciones de apolipoproteína B (ApoB $)^{7}$. Estas partículas de LDL entran rápidamente a través de la pared vascular y pueden ser tóxicas para las células endoteliales, causar mayor producción de factores procoagulantes, ser oxidadas e inmovilizadas más rápidamente por los proteoglicanos presentes en la pared arterial y de esta forma estimular el desarrollo del proceso de aterogénesis.

Por otro lado, los pacientes con DM2 presentan bajas concentraciones de colesterol en las lipoproteínas de alta densidad (HDL-C) y bajos niveles circulantes de la apolipoproteína AI (ApoAI) 7,27. Por lo que es de esperar un efecto perjudicial en el contenido de colesterol en la pared vascular. El transporte reverso de colesterol que realizan las HDL se ve limitado, no sólo por la disminución en su concentración, sino también por procesos como la exposición a proteínas glicosiladas, glicación de la ApoAI y cambios en la composición de las lipoproteínas $^{28}$. Los macrófagos aislados de individuos con bajas concentraciones de HDL-C presentan un fenotipo pro-inflamatorio, lo que podría explicar en parte su contribución al proceso de ateroesclerosis ${ }^{29}$.

Mecanismos de inflamación y aterosclerosis en pa-

\section{cientes diabéticos}

Diferentes estudios permitieron establecer el papel de las células inflamatorias (macrófagos y linfocitos $\mathrm{T}$ ) y mecanismos inflamatorios (liberación de citoquinas) en la patogénesis de la aterosclerosis. Es difícil establecer una relación causa-efecto entre la DM2 y la aterosclerosis en términos de inflamación, pues se sabe que este proceso está implicado en la patogénesis de ambas patologías.

La hiperglicemia y dislipidemia característicos de la DM2 conducen a una respuesta inflamatoria del endotelio. Las respuestas endoteliales clásicas pro ateroscleróticas: expresión de moléculas de adhesión, secreción de quimoquinas y proteínas de coagulación (inhibidor del activador del plasminógeno-1, factor tisular), liberación de mediadores vasoactivos (NO endotelial y bradiquininas) son inducidas o reguladas por estímulos inflamatorios en modelos in vitro e in vivo de diabetes.

Los linfocitos al ser activados por estímulos metabólicos producen señales proinflamatorias importantes para los macrófagos derivados de monocitos y las células de músculo liso vascular. Los macrófagos pueden responder de forma directa a las anormalidades comunes en la DM2 como son la hiperglicemia, ácidos grasos libres e hipertrigliceridemia, aumentando la respuesta inflamatoria. Varios estímulos y procesos celulares están implicados en los efectos de los macrófagos en la aterosclerosis incluyendo el aumento de la formación de células espumo- 
Cuadro 1. Genética en la diabetes mellitus tipo 2 y cardiopatía isquémica Rol de la genética:

La susceptibilidad genética es la probabilidad de presentar una determinada enfermedad, el estudio genético ha aumentado el conocimiento del impacto de los genes en cada enfermedad. En los últimos años se han descubierto varios factores de riesgos genéticos implicados en diabetes y cardiopatía isquémica a través de estudios de asociación del genoma completo (GWAS, en inglés) ${ }^{31,32}$. La DM2 es el resultado de una interacción compleja entre la genética y el medio ambiente, existe un conjunto de loci de susceptibilidad bien identificados, que dan respuesta a un $10 \%$ de la genética y herencia familiar de esta enfermedad. Sin embargo, a través de estudios de segregación familiar, se estableció que la prevalencia entre integrantes de una misma familia es superior a lo esperado en la población general, debido a los genes compartidos entre ellos ${ }^{32}$. El estudio de Framingham fue uno de los primeros estudios en evaluar este riesgo, al establecer que las personas con un padre diabético tenían 3.5 veces mayor riesgo de desarrollar la enfermedad y que este riesgo aumentaba a 6.1, si ambos padres eran diabéticos ${ }^{33}$. Estudios en gemelos monocigóticos y dicigóticos, asumiendo que entre hermanos estuvieron expuestos al mismo ambiente, se mostró que los monocigóticos presentaban mayor riesgo de diabetes si el hermano presentaba la enfermedad 32,34 .

Este tipo de hallazgos han motivado la búsqueda de loci candidatos adicionales que apunten al desarrollo de una experimentación molecular, funcional y terapéutica rigurosa y de esta manera abordar variables individuales, que se relacionen o examinar las vías de señalización moleculares moduladas por estas alteraciones génicas descritas en la DM2.

Genes compartidos:

El estudio reciente realizado por investigadores de la Universidad de Pittsburgh evaluó aquellos genes compartidos entre pacientes con DM2 y cardiopatía isquémica e incluyó un estudio de asociación del genoma completo, en 265,678 sujetos con DM2 y 269,365 sujetos con cardiopatía coronaria. Se identificaron 16 nuevos loci asociados con DM2 y 1 nuevo locus para cardiopatía coronaria. Además se mostró que el aumento del riesgo genético para DM2 confiere un mayor riesgo de cardiopatía coronaria (Tabla 1). Esta interacción entre DM2 y cardiopatía coronaria mostró ser unidireccional, ya que los genes que determinan un mayor riesgo para desarrollar DM2 aumentan el riesgo de cardiopatía coronaria, pero esto no ocurre necesariamente en el sentido inverso.

El análisis del riesgo en conjunto DM2 y cardiopatía coronaria identificó 8 variantes genéticas que colocalizan, algunas previamente ya conocidas para DM2 (TCF7L2, HNF1A, CTRB1, CTRB2), otras variantes para DM2 descritas fueron MIR17HG, CCDC92 y otras ya conocidas por su asociación con el riesgo de cardiopatía coronaria (MRAS, ZC3HC1) (Tabla 2).

Resultó interesante establecer que varios de los loci descubiertos dan cuenta de la biología de la DM2, confirman vías implicadas en esta enfermedad e incluso aún sin ser loci que compartan DM2 y la cardiopatía coronaria, permiten relacionar el daño endotelial y la respuesta inflamatoria característica de ésta relación DM2- cardiopatía coronaria. Por ejemplo, MIR17HG, KL y BCL2L11 están implicados en la sobrevida celular, apoptosis y envejecimiento celular, respectivamente. También se ha mostrado que las variantes genéticas cercanas a KL también se asocian con los niveles de glucosa en ayunas. Por su parte TMEM ${ }^{18}$ está involucrado en la migración celular; HLA-DR5 y CMIP tienen un papel crucial en las respuestas inmunes y participan en diversos trastornos inmunológi$\cos ^{32}$. Finalmente, también se definieron algunos biomarcadores de riesgo, como APOE, cuya variante en pacientes con DM2 se asoció a una disminución de LDL-C (factor de riesgo) y, además, se relacionó directamente con una disminución del riesgo de desarrollo de cardiopatía insquémica $32,35,36$

Estos hallazgos resaltan cómo la genética humana podría ayudar a enfocar futuras investigaciones de estrategias terapéuticas en la DM2 y los prometedores efectos que tendrían sobre los estudios clínicos vasculares.

(Tablas 1 y 2)

\begin{tabular}{|cc|}
\hline Gen & Cromosoma \\
\hline TMEM18 & 2 \\
BCL2L11 & 2 \\
TMEM155 & 4 \\
PHF15 & 5 \\
SLC22A3 & 6 \\
BRAF & 7 \\
KCNU1 & 8 \\
RNF6 & 13 \\
KL & 13 \\
MIR17HG & 13 \\
ITFG3 & 16 \\
HLA-DRB5 & 6 \\
PLEKHA1 & 10 \\
CMIP & 16 \\
CCDC92 & 12 \\
\hline
\end{tabular}

Tabla. 1 Nuevos loci asociados a diabetes mellitus 2.

\begin{tabular}{|cc|}
\hline Gen & \multicolumn{1}{c|}{ Cromosoma } \\
\hline \multicolumn{2}{|c|}{ Loci establecidos DM2-Cl } \\
\hline TCF7L2 & 10 \\
\hline I27L & 12 \\
\hline CTRB1/2 & 16 \\
\hline MRAS & 3 \\
\hline ZC3HC1 & 7 \\
\hline \multicolumn{2}{|c|}{ Loci nuevos DM2-CI } \\
\hline MIR17HG & 13 \\
\hline CCDC92 & 12 \\
\hline Alelos opuestos de riesgo para DM2-CI \\
\hline APOE & 19 \\
\hline
\end{tabular}

Tabla 2. Loci asociados a diabetes mellitus 2 (DM2) y cardiopatía isqué$\operatorname{mica}(\mathrm{CI})$. 
sas, liberación de metaloproteinas de la matriz, secreción de factores de crecimiento y citoquinas. Estos efectos muestran la conexión entre resistencia a la insulina, inflamación y aterosclerosis (Figura 4).

Un regulador central de la inflamación es NF-kB, un complejo transcripcional activado por varios estímulos, incluyendo citoquinas, LDL oxidado, lipopolisacárido y estrés oxidativo. NF-kB regula la modificación oxidativa de las LDL, la expresión de citoquinas y quimoquinas, crecimiento y diferenciación de los macrófagos, apoptosis y proliferación celular de las células del músculo liso vascular. Tanto NF-kB, sus proteínas regulatorias (p.ej. IkB) y sus blancos se han implicado en la sensibilidad a la insulina y la aterosclerosis. NF-kB se activa por factores comúnmente alterados en DM2, incluyendo ácidos grasos libres, glucosa, AGEs y algunos receptores tipo toll (TLR).

Algunos blancos regulados por NF-kB se asocian al desarrollo de la aterosclerosis diabética, incluyendo TNF $\alpha$. Estos incrementan la resistencia a la insulina, TLRs y resistina. Los efectos anti-inflamatorios y anti-ateroscleróticos de PPAR $\gamma$ se deben probablemente a la inhibición del NF-kB.

Se han propuesto diversos mecanismos que explican como la glucosa genera daño celular y subsecuente inflamación. Las células frente a un incremento excesivo de las concentraciones intracelulares de glucosa activan vías de daño celular e inflamación. Estos mecanismos incluyen la activación de la PKC y producción de polioles, los que promueven estrés oxidativo, e incrementan la activación de la vía de las hexosaminas, mayor incremento en las especies reactivas de oxígeno y estrés mitocondrial. Diversos grupos de investigación proponen que el estrés del retículo endoplásmico (RE) es un evento crucial en la promoción de inflamación. El estrés de RE y la respuesta a proteínas mal plegadas (UPR) puede ser inducido por hipoxia, hiperglicemia y aumento de las concentraciones de ácidos grasos. El estrés de RE, presente en el hígado y el tejido adiposo, puede activar vías que conducen a oxidación e inflamación, y han sido implicados tanto en DM2 como aterosclerosis.

\section{Manejo clínico y terapias convencionales}

En los últimos años se ha optimizado el manejo clínico de la diabetes mediante la introducción de nuevas opciones terapéuticas, sin embargo, es importante valorar el efecto del tratamiento hipoglicemiante sobre el sistema cardiovascular, debido a la alta morbi-mortalidad asociada en los pacientes diabéticos. Si bien la

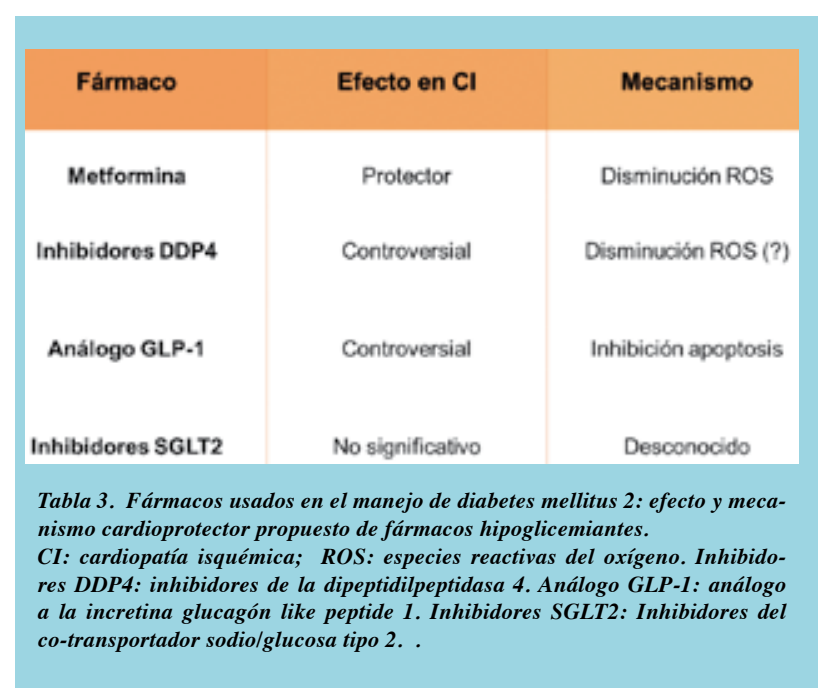

disminución de la glucosa plasmática reduce de por sí el daño endotelial algunos fármacos presentan efectos adicionales (Tabla 3 ).

La metformina, fármaco de primera línea en el tratamiento de la diabetes, ha mostrado disminuir significativamente los eventos de infarto al miocardio aún a los 10 años de seguimiento ${ }^{37,38}$. Se postula que el mecanismo de acción es la disminución de ROS, lo que disminuiría el daño endotelial, característica principal en la aterosclerosis 39 .

Los inhibidores de DDP-4 (gliptinas) reducen los triglicéridos y la presión arterial, factores de riesgo cardiovascular. Además, se constató en un modelo murino de isquemia miocárdica que disminuye el tamaño de infarto y mejora la funcionalidad miocárdica ${ }^{40}$. Sin embargo, en los ensayos clínicos SAVOR-TIMI, EXAMINE Y TECOS, las gliptinas no mostraron ser superiores al tratamiento con otros fármacos ${ }^{41-43}$. La evidencia actual sobre el efecto cardiovascular de los análogos de GLP-1 no es concluyente debido a que si bien ha mostrado poseer efectos benéficos en estudios animales y acciones pleiotrópicas que reducen el riesgo cardiovascular $^{44,45}$, existe discordancia en los ensayos clínicos. El estudio (ELIXA) que utilizó lixisenatide no observó beneficios. Sin embargo en el estudio (LEADER), liraglutide disminuyó el riesgo de muerte de causa cardiovascular, eventos de infarto del miocardio de forma significativa ${ }^{46,47}$.

El estudio EMPA-REG evidenció la disminución de muerte por causa cardiovascular del inhibidor SGTL2 empaglifozina. Sin embargo, no hubo diferencias significativas en eventos de infarto del miocardio. Aunque se desconoce el mecanismo de acción de este fármaco se 
- Estudios recientes han identificado 16 nuevos genes asociados a diabetes mellitus tipo 2.

- Análisis del riesgo conjunto de DM2 y cardiopatía coronaria identificó 8 variantes genéticas comunes para ambas patologías y dos nuevos loci que constituyen blancos moleculares para el tratamiento de DM2 y disminución de eventos cardiovasculares.

- La asociación bidireccional entre la disfunción endotelial y DM2 modulada por un componente génico apunta a realizar estudios que permitan establecer el impacto epigenético existente sobre la regulación de la transcripción de genes pro-inflamatorios, generadores de ROS y responsables de daño endotelial por estrés oxidativo.

- El daño endotelial característico de pacientes DM2 y la tipificación de nuevos genes que modulen la expresión de proteínas, dan oportunidad a la caracterización de nuevos biomarcadores que se asocien directamente con incidentes cardiovasculares y permitan la prevención de estas complicaciones.

- La identificación de nuevos genes y polimorfismos en la DM2 y cardiopatía coronaria da paso a investigaciones sobre vías moleculares asociadas a envejecimiento celular, apoptosis, migración y respuesta inflamatoria endotelial.

Relevancia

La diabetes mellitus tipo 2 (DM2), la forma más común de la enfermedad, puede permanecer indetectada por muchos años y su diagnóstico a menudo se realiza tardíamente. Por lo tanto, se trata esta enfermedad en una etapa avanzada cuando las complicaciones vasculares ya se han producido en la mayoría de los pacientes, estas son de carácter irreversible y la principal causa de mortalidad en estos pacientes.

Dado este escenario, una mejor comprensión de los mecanismos y modulación génica que subyacen a la enfermedad vascular diabética es obligatoria porque proporcionará nuevos enfoques y uso de nuevas tecnologías como terapia génica y anticuerpos monoclonales que prevengan o retrasen el desarrollo de sus complicaciones.

postula que puede deberse a sus efectos hemodinámicos, disminución de la presión arterial y mejoría de la función cardiovascular.

\section{Nuevas opciones: Terapia génica y blancos moleculares}

La investigación de las vías y procesos biológicos presentes tanto en la DM2 y cardiopatía isquémica podría abrir la puerta a nuevos blancos terapéuticos.

El descubrimiento de genes asociados a ambas enfermedades supone la posibilidad de identificación de mecanismos moleculares adicionales, lo que ofrece nuevas alternativas terapéuticas. La terapia génica representa el manejo ideal de distintas enfermedades, se basa en la modulación de genes anómalos o ausentes de manera individualizada. Sin embargo, aún existen dificultades técnicas para la aplicación y uso de esta prometedora herramienta. No obstante, la identificación de nuevos loci involucra blancos moleculares que pueden ser abordados con medi- camentos ya existentes, éstos incluirían al Icosapento, un ácido graso poliinsaturado que se encuentra en el aceite de pescado, el cual es un agonista de FFAR1 y PPARG ${ }^{48}$ e inhibidor de COX1/ COX2 $2^{48,49} y$, a través de ensayos clínicos (ANCHOR), ha mostrado disminuir factores de riesgo de éste círculo vicioso formado entre la DM2 y el daño endotelial (triglicéridos y HDL-C) ${ }^{49}$.

También se han identificado polimorfismos que correlacionan con la cardiopatía coronaria subclínica en familias con DM2. Específicamente, la variación genética de los genes PON1, PON2 y LPL, los cuales parecen contribuir a la calcificación arterial, ha dado paso a investigaciones que ahonden las vías moduladas por estos genes, las cuales podrían ser dianas terapéuticas útiles para individuos portadores de alelos de riesgo ${ }^{35}$.

Otro gen candidato plausible es el FABP4, el cual codifica la proteína de unión a ácidos grasos de adipocitos, también conocida como aP2. Los modelos de ratones deficientes en aP2 muestran protección contra la aterosclerosis y un fenotipo antidiabético ${ }^{50}$. Además, la inhibición de aP2 reduce la aterosclerosis, los niveles de glucosa e insulina y los triglicéridos en estudios in vivo ${ }^{51}$; por tanto se han iniciado investigaciones que abordan la inhibición de esta vía a través de un anticuerpo monoclonal, lo cual constituiría un potente recurso en el manejo dual tanto de la hiperglicemia como aterosclerosis en pacientes con $\mathrm{DM} 2^{52}$ 


\section{Referencias}

1. GUARIGUATA L, WHITING DR, HAMBLETON I, BEAGLEY J, LINNENKAMP U, SHAW JE. Global estimates of diabetes prevalence for 2013 and projections for 2035. Diabetes Research and Clinical Practice. 2014; 103: 137-149.

2. WORLD HEALTH Organization. World health statistics 2015. 2016. http://www.who.int/gho/epidemic_diseases/meningitis/en/.

3. AMERICAN DIABETES ASSOCIATION. Classification and diagnosis of diabetes. Diabetes Care. 2017; 40: S11-S24.

4. BLOOMGARDEN ZT. Cardiovascular disease in diabetes. Diabetes Care. 2010;33:e49-54.

5. REDDY KS. Global Burden of Disease Study 2015 provides GPS for global health 2030. The Lancet. 2016; 388: 14481449.

6. FINEGOLD JA, ASARIA P, FRANCIS DP. Mortality from ischaemic heart disease by country, region, and age: statistics from World Health Organisation and United Nations. International Journal of Cardiology. 2013; 168: 934-945.

7. MAZZONE T, CHAIT A, PLUTZKY J. Cardiovascular disease risk in type 2 diabetes mellitus. Insights from mechanistic studies. The Lancet. 2008; 371: 1800-1809.

8. SHAHAB U, AHMAD MK, MAHDI AA, WASEEM M, ARIF $B$, MOINUDDIN, et al. The receptor for advanced glycation end products: A fuel to pancreatic cancer. Seminars in Cancer Biology. 2017.

9. FRANKEL DS, MEIGS JB, MASSARO JM, WILSON PWF, O'DONNELL CJ, D'AGOSTINO RB, et al. Von Willebrand factor, type 2 diabetes mellitus, and risk of cardiovascular disease: the Framingham offspring study. Circulation. 2008; 118: 2533-2539.

10. HUXLEY R, BARZI F, WOODWARD M. Excess risk of fatal coronary heart disease associated with diabetes in men and women: meta-analysis of 37 prospective cohort studies. BMJ. 2006; 332: 73-78.

11. SARWAR N, SATTAR N, GUDNASON V, DANESH J. Circulating concentrations of insulin markers and coronary heart disease: a quantitative review of 19 Western prospective studies. European Heart Journal. 2007; 28: 2491-2497.

12. CARVAJAL CARVAJAL C. Productos finales de glicación (AGES) y la nefropatía diabética. Medicina Legal de Costa
Rica. 2015; 32: 154-160.

13. SARAFIDIS PA, LAZARIDIS AA, RUIZ-HURTADO G, RUILOPE LM. Blood pressure reduction in diabetes: lessons from ACCORD, SPRINT and EMPA-REG OUTCOME. Nature Reviews. Endocrinology. 2017; 13: 365-374.

14. HIGASHI Y, NOMA K, YOSHIZUMI M, KIHARA Y. Endothelial function and oxidative stress in cardiovascular diseases. Circulation Journal. 2009; 73: 411-418.

15. OMI H, OKAYAMA N, SHIMIZU M, FUKUTOMI T, IMAEDA K, OKOUCHI M, et al. Statins inhibit high glucose-mediated neutrophil-endothelial cell adhesion through decreasing surface expression of endothelial adhesion molecules by stimulating production of endothelial nitric oxide. Microvascular Research. 2003; 65: 118-124.

16. ZANNAD F. Implications of the ADVANCE study for clinical practice. Journal of Hypertension.2008; 26: S29-32.

17. MOHAMMEDI K, WOODWARD M, HIRAKAWA Y, ZOUNGAS S, COLAGIURI S, HAMET P, et al. Presentations of major peripheral arterial disease and risk of major outcomes in patients with type 2 diabetes: results from the ADVANCE-ON study. Cardiovascular Diabetology. 2016; 15: 129.

18. COHEN RA. Role of nitric oxide in diabetic complications. American Journal of Therapeutics. 2005; 12: 499-502.

19. YAN SD, SCHMIDT AM, ANDERSON GM, ZHANG J, BRETT J, ZOU YS, et al. Enhanced cellular oxidant stress by the interaction of advanced glycation end products with their receptors/binding proteins. The Journal of Biological Chemistry. 1994; 269: 9889-9897.

20. KIM J-A, MONTAGNANI M, KOH KK, QUON MJ. Reciprocal relationships between insulin resistance and endothelial dysfunction: molecular and pathophysiological mechanisms. Circulation. 2006; 113: 1888-1904.

21. PEIRÓ C, LORENZO Ó, CARRARO R, SÁNCHEZ-FERRER CF. IL- $1 \beta$ inhibition in cardiovascular complications associated to diabetes mellitus. Frontiers in Pharmacology. 2017; 8: 363.

22. DASU MR, DEVARAJ S, JIALAL I. High glucose induces IL-1beta expression in human monocytes: mechanistic insights. American Journal of Physiology. Endocrinology and Metabolism. 2007; 293: E337-46. 
23. VAN SLOTEN TT, HENRY RMA, DEKKER JM, NIJPELS G, UNGER T, et al. Endothelial dysfunction plays a key role in increasing cardiovascular risk in type 2 diabetes: the Hoorn study. Hypertension 2014; 64: 1299-1305.

24. SHIN HK, KIM YK, KIM KY, LEE JH, HONG KW. Remnant lipoprotein particles induce apoptosis in endothelial cells by $\mathrm{NAD}(\mathrm{P}) \mathrm{H}$ oxidase-mediated production of superoxide and cytokines via lectin-like oxidized low-density lipoprotein receptor-1 activation: prevention by cilostazol. Circulation. 2004; 109: 1022-1028.

25. TING HJ, STICE JP, SCHAFF UY, HUI DY, RUTLEDGE JC, KNOWLTON AA, et al. Triglyceride-rich lipoproteins prime aortic endothelium for an enhanced inflammatory response to tumor necrosis factor-alpha. Circulation Research. 2007; 100: $381-390$.

26. SILVERMAN MG, FERENCE BA, IM K, WIVIOTT SD, GIUGLIANO RP, GRUNDY SM, et al. Association between lowering LDL-C and cardiovascular risk reduction among different therapeutic interventions: A systematic review and meta-analysis. JAMA. 2016; 316: 1289-1297.

27. MOORADIAN AD, HAAS MJ, WONG NCW. Transcriptional control of apolipoprotein A-I gene expression in diabetes. Diabetes. 2004; 53: 513-520.

28. KASHYAP SR, OSME A, ILCHENKO S, GOLIZEH M, LEE $\mathrm{K}$, WANG S, et al. Glycation reduces the stability of apoAI and increases HDL dysfunction in diet-controlled type 2 diabetes. The Journal of Clinical Endocrinology and Metabolism. 2018; 103: 388-396.

29. XU L, WANG Y-R, LI P-C, FENG B. Advanced glycation end products increase lipids accumulation in macrophages through upregulation of receptor of advanced glycation end products: increasing uptake, esterification and decreasing efflux of cholesterol. Lipids in Health and Disease. 2016; 15: 161.

30. PANENI F, BECKMAN JA, CREAGER MA, COSENTINO F. Diabetes and vascular disease: pathophysiology, clinical consequences, and medical therapy: part I. European Heart Journal. 2013; 34: 2436-2443.

31. MENZAGHI C, TRISCHITTA V, DORIA A. Genetic influences of adiponectin on insulin resistance, type 2 diabetes, and cardiovascular disease. Diabetes. 2007; 56: 1198-1209.

32. ZHAO W, RASHEED A, TIKKANEN E, LEE J-J, BUTTERWORTH AS, HOWSON JMM, et al. Identification of new susceptibility loci for type 2 diabetes and shared etiological pathways with coronary heart disease. Nature Genetics. 2017; 49: $1450-1457$.
33. MAHMOOD SS, LEVY D, VASAN RS, WANG TJ. The Framingham Heart Study and the epidemiology of cardiovascular disease. A historical perspective. The Lancet. 2014; 383: 999 1008 .

34. TATTERSALL RB, PYKE DA. Diabetes in identical twins. The Lancet 1972; 2: 1120-1125.

35. BURDON KP, LANGEFELD CD, BECK SR, WAGENKNECHT LE, CARR JJ, FREEDMAN BI, et al. Association of genes of lipid metabolism with measures of subclinical cardiovascular disease in the Diabetes Heart Study. Journal of Medical Genetics. 2005; 42: 720-724.

36. SATTAR N, PREISS D, MURRAY HM, WELSH P, BUCKLEY BM, CRAEN AJM et al. Statins and risk of incident diabetes. A collaborative meta-analysis of randomised statin trials. The Lancet. 2010;375:735-742.

37. HOLMAN RR, PAUL SK, BETHEL MA, MATTHEWS DR, NEIL HAW. 10-year follow-up of intensive glucose control in type 2 diabetes. The New England Journal of Medicine. 2008; 359: 1577-1589.

38. EFFECT OF INTENSIVE BLOOD-GLUCOSE control with metformin on complications in overweight patients with type 2 diabetes (UKPDS 34). UK Prospective Diabetes Study (UKPDS) Group. The Lancet. 1998; 352: 854-865.

39. FORETZ M, GUIGAS B, BERTRAND L, POLLAK M, VIOLLET B. Metformin: from mechanisms of action to therapies. Cell Metabolism. 2014; 20: 953-966.

40. KUBOTA A, TAKANO H, WANG H, HASEGAWA H, TADOKORO H, HIROSE M, et al. DPP-4 inhibition has beneficial effects on the heart after myocardial infarction. Journal of Molecular and Cellular Cardiology. 2016; 91: 72-80.

41. GREEN JB, BETHEL MA, ARMSTRONG PW, BUSE JB, ENGEL SS, GARG J, et al. Effect of sitagliptin on cardiovascular outcomes in type 2 diabetes. The New England Journal of Medicine. 2015; 373: 232-242.

42. WHITE WB, CANNON CP, HELLER SR, NISSEN SE, BERGENSTAL RM, BAKRIS GL, et al. Alogliptin after acute coronary syndrome in patients with type 2 diabetes. The New England Journal of Medicine. 2013; 369: 1327-1335.

43. UDELL JA, BHATT DL, BRAUNWALD E, CAVENDER MA, MOSENZON O, STEG PG, et al. Saxagliptin and cardiovascular outcomes in patients with type 2 diabetes and moderate or severe renal impairment: observations from the SAVOR-TIMI 53 trial. Diabetes Care. 2015; 38: 696-705.

44. ZIMMERMAN RS, HOBBS TM, WELLS BJ, KONG SX, 
KATTAN MW, Bouchard J, et al. Association of glucagon-like peptide-1 receptor agonist use and rates of acute myocardial infarction, stroke and overall mortality in patients with type 2 diabetes mellitus in a large integrated health system. Diabetes, Obesity \& Metabolism. 2017; 19: 1555-1561.

45. DAVIDSON MH. Cardiovascular effects of glucagonlike peptide-1 agonists. The American Journal of Cardiology. 2011; 108: 33B-41B.

46. MARSO SP, DANIELS GH, BROWN-FRANDSEN K, KRISTENSEN P, MANN JFE, NAUCK MA, et al. Liraglutide and cardiovascular outcomes in type 2 diabetes. The New England Journal of Medicine. 2016; 375: 311-322.

47. PFEFFER MA, CLAGGETT B, DÍAZ R, DICKSTEIN K, GERSTEIN HC, KØBER LV, et al. Lixisenatide in patients with type 2 diabetes and acute coronary syndrome. The New England Journal of Medicine. 2015; 373: 2247-2257.

48. LAW V, KNOX C, DJOUMBOU Y, JEWISON T, GUO AC, LIU Y, et al. DrugBank 4.0: shedding new light on drug metabolism. Nucleic Acids Research. 2014; 42: D1091-7.
49. BALLANTYNE CM, BRAECKMAN RA, BAYS HE, KASTELEIN JJ, OTVOS JD, STIRTAN WG, et al. Effects of icosapent ethyl on lipoprotein particle concentration and size in statin-treated patients with persistent high triglycerides (the ANCHOR Study). Journal of Clinical Lipidology. 2015; 9: $377-383$.

50. BOORD JB, MAEDA K, MAKOWSKI L, BABAEV VR, FAZIO S, LINTON MF, et al. Adipocyte fatty acid-binding protein, aP2, alters late atherosclerotic lesion formation in severe hypercholesterolemia. Arteriosclerosis, Thrombosis, and Vascular Biology. 2002; 22: 1686-1691.

51. FURUHASHI M, TUNCMAN G, GÖRGÜN CZ, MAKOWSKI L, ATSUMI G, VAILLANCOURT E, et al. Treatment of diabetes and atherosclerosis by inhibiting fatty-acid-binding protein aP2. Nature. 2007;447:959-965.

52. BURAK MF, INOUYE KE, WHITE A, LEE A, TUNCMAN G, CALAY ES, et al. Development of a therapeutic monoclonal antibody that targets secreted fatty acid-binding protein aP2 to treat type 2 diabetes. Science Translational Medicine. 2015; 7: 319ra205. 